\title{
Vitesse du son dans les sédiments marins durant les premiers stades du tassement
}

\author{
Sound velocity in marine sediments \\ during the early stages of settling
}

\author{
P. GUILlAUME, P. MORLIER, C. VIGUIER
}

Laboratoire d'Etudes et de Recherches en Géotechniques et Géophysique Appliquée*

Rev. Franç. Géotech. n 57, pp. $49-57$ (octobre 1991)

\section{Résumé}

La vitesse du son est mesurée dans des sédiments marins lors d'une étude expérimentale du tassement. Durant la phase de sédimentation, la vitesse du son diminue légèrement, puis lors de la phase de compaction elle s'accroît avec la charge et la réduction de porosité (modèles de WOOD (1941) et de NOBES (1989)). Le suivi du phénomène met en évidence le réarrangement graduel de la structure des particules et l'apparition de la rigidité pour une porosité seuil (environ 0,70 ). La différence de comportement acoustique entre les matériaux argileux et carbonatés résulte essentiellement de la granulométrie qui contrôle la réorganisation des grains.

\begin{abstract}
An experimental study which measure the sound velocity during the settling of marine sediments is presented. In the first step the sound velocity slightly decrease during decantation stage, in the second step it increases with loading and porosity reduction during compaction stage (WOOD (1941) and NOBES (1989) models). The follow of the process shows the progressive rearrangement of particules structure and the acquisition of rigidity appears at a threshold porosity (about 0.70 ). Acoustical behaviour of argileous and calcareous sediments mainly differs owing to grain-size distribution which control the particles reorganization.
\end{abstract}




\section{INTRODUCTION}

Des sédiments marins carottés lors des missions océanographiques Caracolante 1 et 2 réalisées par l'Institut Géologique du Bassin d'Aquitaine en 1981 et 1985 dans le domaine Caraibe (Bassin Vénézuélien, Prisme de la Barbade et littoral de la Martinique), ont donné lieu à une analyse sédimentologique et géotechnique, dans le but de préciser les interactions entre le milieu de dépôt et les caractéristiques géomécaniques. Les résultats obtenus ont montré d'une part l'importance de la composition granulométrique et minéralogique dans le comportement géotechnique de matériaux (notamment de la présence d'une teneur en carbonate supérieure à $40 \%$ ) et d'autre part l'influence des processus de dépôt marquée à travers l'arrangement des particules (GUILLAUME, 1990). Ceci nous a conduit à examiner les mécanismes régissant le mode de * fabrication " des sols argileux et/ou carbonatés, à la fois sur des sédiments naturels et sur des sédiments reconstitués. Nous avons donc réalisé une étude expérimentale de la sédimentation et des premiers stades de la compaction simulant l'enfouissement progressif des sédiments. L'utilisation d'une méthode d'investigation indirecte telle que la mesure de la vitesse du son, permet alors de visualiser les processus mis en jeu lors du passage fluide - suspension - solide, sans perturber la structure du matériau. On peut ainsi suivre l'évolution des relations entre la phase liquide et les particules solides durant la sédimentation et le début de la compaction.

\section{RAPPELS THÉORIQUES}

Un matériau sédimentaire sous-marin constitue un milieu où les particules sont plus ou moins en contact dans un fluide. Au point de vue de son comportement acoustique, il est considéré, selon sa porosité $(n)$, soit comme une suspension de particules ( $n>0,5$ ), soit comme un solide poreux ( $n<0,3$ ) où les grains ne sont plus indépendants les uns des autres. Les sédiments que nous avons analysés possédant une porosité élevée, les théories concernant les suspensions et les agrégats granulaires sont mieux adaptées que celles des milieux poreux.

Dans les suspensions et émulsions, la vitesse du son évolue selon une loi simple de mélange à deux composantes (WOOD, 1941) :

$$
\begin{aligned}
& V_{L}= \\
& {\left[\frac{1}{\left[\rho_{w} \cdot n+\rho_{s}(1-n)\right]\left[k_{w} \cdot n+k_{s}(1-n]\right.}\right]^{1 / 2}}
\end{aligned}
$$

la masse volumique de la solution : $\rho=\rho_{\mathrm{w}} \cdot \mathrm{n}+$ $\rho_{5}(1-n)$, avec $\rho_{w}$ la masse volumique du liquide, $\rho_{5}$ la masse volumique des grains et $\mathrm{n}$ la porosité du milieu ( $\left.\mathrm{n}=\mathrm{V}_{\text {eau }} / \mathrm{V}_{\text {total }}\right)$ et la compressibilité du système : $\mathrm{k}=\mathrm{k}_{\mathrm{w}} \cdot \mathrm{n}+\mathrm{k}_{\mathrm{s}}(1-\mathrm{n})$, avec $\mathrm{k}_{\mathrm{w}}$ la compressibilité du liquide et $\mathrm{k}_{\mathrm{s}}$ la compressibilité du solide.
Or d'une manière générale, les mesures expérimentales de célérité des ondes sur des sédiments naturels donnent des valeurs supérieures à celles de WOOD (GREFFARD, 1969; HAMILTON et al., 1982 ; BACHMAN, 1985 ; ORSI et DUNN, 1990...). LAUGHTON (1957) à partir de l'étude de la compaction de divers sédiments naturels introduit pour sa part le concept d'incompressibilité de structure $\mathrm{k}=$ $\mathrm{k}_{\mathrm{w}} \cdot \mathrm{n}+\mathrm{k}_{\mathrm{s}}(1-\mathrm{n})+\mathrm{k}_{\mathrm{c}}$, et SHUMWAY (1960) suppose un coefficient de rigidité $\mu$.

$$
\begin{aligned}
& V_{L}=\left[\frac{1}{\left[\rho_{w} \cdot n+\rho_{s}(1-n)\right]}\right. \\
& \left.\left(\frac{1}{\left.k_{w} \cdot n+k_{s}(1-n)\right]}+\frac{4}{3} \mu\right)\right]^{1 / 2}
\end{aligned}
$$

Des théories sur les milieux poreux saturés, nous retiendrons le modèle de WYLLIE et al. (1956) qui prend en compte le comportement propre à chaque phase, liquide ou solide, à travers la relation suivante:

$$
\frac{1}{V_{L}}=\frac{n}{V_{w}}+\frac{1-n}{V_{s}}
$$

$\mathrm{n}$ : porosité ;

$\mathrm{V}_{\mathrm{L}}$ : vitesse dans la solution;

$V_{W}$ : vitesse dans le fluide ;

$\mathrm{V}_{\mathrm{S}}$ : vitesse dans les grains.

NOBES (1989) propose ensuite une combinaison des modèles de WOOD et de WYLLIE qui considère le fluide comme une suspension et les particules comme des matériaux poreux :

$$
\frac{1}{V_{L}}=\frac{n}{V_{\text {Wood }}}+\frac{1-n}{V_{\text {Wyllie }}}
$$

\section{MÉTHODOLOGIE}

\subsection{Le matériel}

Un œdomètre est équipé d'un système de mesure de la vitesse des ondes acoustiques, permettant une double utilisation en colonne de décantation et cellule cedométrique. Dans cette cellule de mesure de $50 \mathrm{~mm}$ de diamètre et $20 \mathrm{~mm}$ de hauteur, deux capteurs ultrasoniques sont diamétralement opposés dans un plan horizontal (fig. 1). Les transducteurs utilisés sont des céramiques piézoélectriques cylindriques de fréquence $300 \mathrm{kHz}$ vibrant dans le sens de l'épaisseur. La distance entre les deux transducteurs étant constante, on peut déterminer à chaque instant la célérité des ondes longitudinales à travers le matériau. La cellule est surmontée d'un tube en plexiglass de $50 \mathrm{~cm}$ de haut pour l'essai de sédimentation ou de $2,5 \mathrm{~cm}$ pour l'essai de compaction.

Lors de la phase de sédimentation, l'appareil est rempli d'eau de salinité de $35 \%$ pour l'étalonnage des mesures de vitesse du son. Puis les particules sont mises en dispersion, la hauteur du floculat ainsi que la célérité des ondes longitudinales à la base de l'éprouvette sont mesurées en fonction du temps (fig. 2). L'essai 


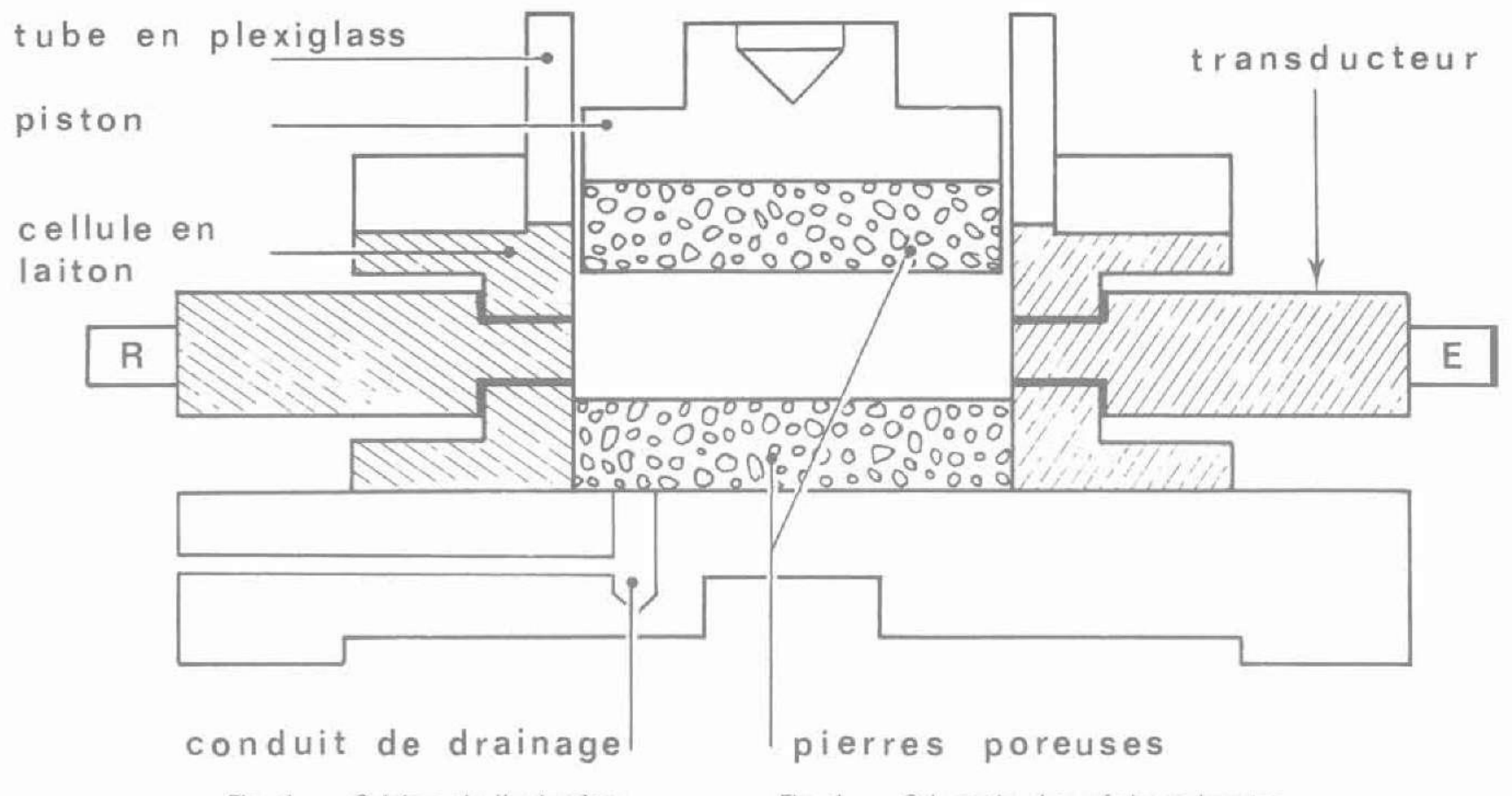

Fig. 1. - Schéma de l'céomètre.

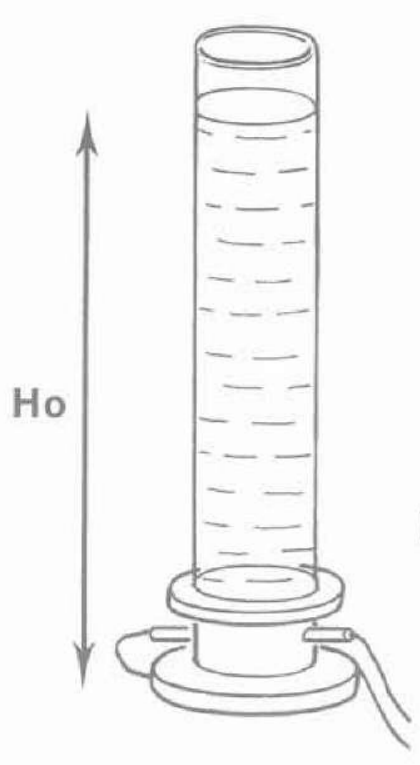

instant To

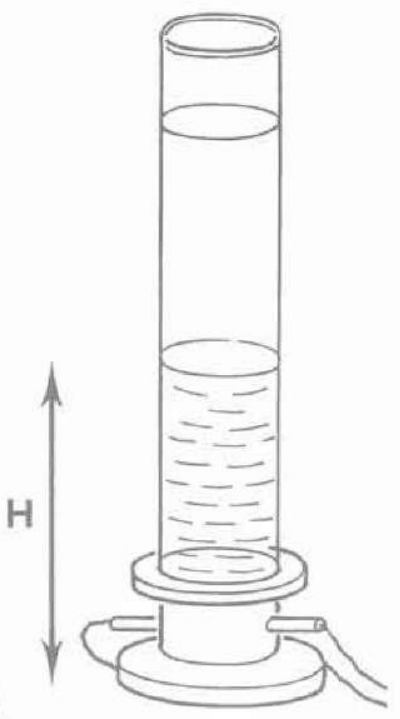

instant T

Fig. 2. - Schéma de mesure du tassement.

Fig. 2. - Diagram of the settling measurement

finit quand la hauteur du dépôt atteint la stabilité après une durée de l'essai qui dépend de la granulométrie du matériau: une journée pour les sables, une semaine pour les échantillons argileux. L'évaluation du tassement se fait par mesure de la hauteur relative de la solution (MIGNIOT, 1968) :

$$
T(\%)=\frac{H}{H_{0}} \times 100
$$

$\mathrm{T}$ : tassement (\%) ;

$\mathrm{H}$ : hauteur de la solution ;

$\mathrm{H}_{\mathrm{o}}$ : hauteur initiale de la solution.

Fig. 1. - Schematic view of the cedometer.

L'essai œedométrique se déroule en suivant deux cycles charge-décharge $(\Delta \sigma / \sigma=1$, durée de charge $24 \mathrm{~h}$, contrainte maximale $2 \mathrm{MPa}$ ). La durée de l'essai complet est d'environ 15 jours.

\subsection{Les sédiments}

Les matériaux étudiés sont des échantillons naturels de granulométrie et de teneur en carbonate variées provenant du domaine Caraibe ainsi que des mélanges réalisés à partir de sédiments argileux et de particules carbonatées. Ils se répartissent en trois classes (leurs caractéristiques sont représentées sur le tableau 1) :

- sables carbonatés $\left(\mathrm{CaCO}_{3} \approx 70 \%\right)$ : SC1-SC2 ; - silts sableux carbonatés $\left(\mathrm{CaCO}_{3} \approx 60 \%\right)$ : SS1SS2-SS3 ;

- argiles silteuses $\left(\mathrm{CaCO}_{3}: 25-35 \%\right)$ : AS1-AS2 AS3-AS4).

\section{DESCRIPTION DU PHÉNOMÈNE DU TASSEMENT}

Durant la phase de sédimentation, la hauteur relative $\mathrm{H} / \mathrm{H}_{\circ}$ en fonction du logarithme du temps (fig. 3) se traduit par une courbe sigmoïde classique plus ou moins accentuée selon la granulométrie du matériau et sa concentration initiale (MIGNIOT, 1968). Le tassement par décantation est complété par l'essai œdométrique. Les deux comportements à la compaction représentés sont typiques de la granulométrie et de la nature des deux catégories de matériaux fins testés (fig. 4). Les échantillons argileux (AS4) possèdent un comportement de type "normal " et celui des silts carbonatés (SS3) est de type « fragile " caractérisé par la rupture des particules (GUILLAUME, 1990). 
Tableau 1. - Caractéristiques des sédiments.

Sa : teneur en sables (\%); si : teneur en silts (\%); Ar; teneur en argiles (\%); $\mathrm{CaCO}_{3}$ : teneur en carbonates $(\%)$; $C_{\text {org }}$ : teneur en carbone organique (\%); ARGILES: pourcentage des différents minéraux argileux dans la fraction $<2 \mu \mathrm{m}\left(\mathrm{Sm}\right.$ : smectites : 1 : illite; $K$ : kaolinite; $C$ : chlorite); $\rho_{s}:$ masse volumique de la phase solide $\left(T / \mathrm{m}^{3}\right) ; W_{L}$ : limite de liquidité $(\%) ; W_{p}$ : limite de plasticité $(\%) ; I P$ : indice de plasticité ; IA : indice d'activité.

\begin{tabular}{|c|c|c|c|c|c|c|c|c|c|c|c|c|c|c|}
\hline Echantillon & \multicolumn{3}{|c|}{$\begin{array}{l}\text { Granulométrie } \\
\mathrm{Sa} \quad \mathrm{si} \quad \mathrm{Ar}\end{array}$} & $\mathrm{CaCO}_{3}$ & $\mathrm{C}_{\text {org }}$ & $\mathrm{Sm}$ & \multicolumn{2}{|c|}{ Argiles } & C & $\rho_{\mathrm{S}}$ & \multicolumn{4}{|c|}{ Limites d'Atterberg } \\
\hline $\begin{array}{c}\text { sables carbonatés } \\
\text { SC1 } \\
\text { SC } 2\end{array}$ & $\begin{array}{l}84 \\
87\end{array}$ & $\begin{array}{l}11 \\
10\end{array}$ & $\begin{array}{l}5 \\
3\end{array}$ & $\begin{array}{l}68 \\
72\end{array}$ & & 36 & 21 & 33 & 11 & 2,60 & & & & \\
\hline $\begin{array}{c}\text { silts sableux } \\
\text { SS1 } \\
\text { SS2 } \\
\text { SS3 }\end{array}$ & $\begin{array}{l}69 \\
33 \\
33\end{array}$ & $\begin{array}{l}20 \\
45 \\
47\end{array}$ & $\begin{array}{l}11 \\
22 \\
20\end{array}$ & $\begin{array}{l}60 \\
57 \\
68\end{array}$ & 0,53 & 23 & 28 & 37 & 12 & $\begin{array}{l}2,55 \\
2,57\end{array}$ & 73,2 & 29,2 & 44 & 2,0 \\
\hline $\begin{array}{c}\text { argiles silteuses } \\
\text { AS1 } \\
\text { AS2 } \\
\text { AS3 } \\
\text { AS4 }\end{array}$ & $\begin{array}{r}29 \\
11 \\
8 \\
9\end{array}$ & $\begin{array}{l}29 \\
31 \\
34 \\
31\end{array}$ & $\begin{array}{l}42 \\
58 \\
58 \\
60\end{array}$ & $\begin{array}{l}35 \\
33 \\
28 \\
25\end{array}$ & $\begin{array}{l}0,19 \\
0,18\end{array}$ & 40 & 20 & 30 & 10 & 2,56 & $\begin{array}{r}92,2 \\
124,8\end{array}$ & $\begin{array}{l}38,2 \\
38,7\end{array}$ & $\begin{array}{l}60,4 \\
86,1\end{array}$ & $\begin{array}{l}1,5 \\
1,4\end{array}$ \\
\hline
\end{tabular}

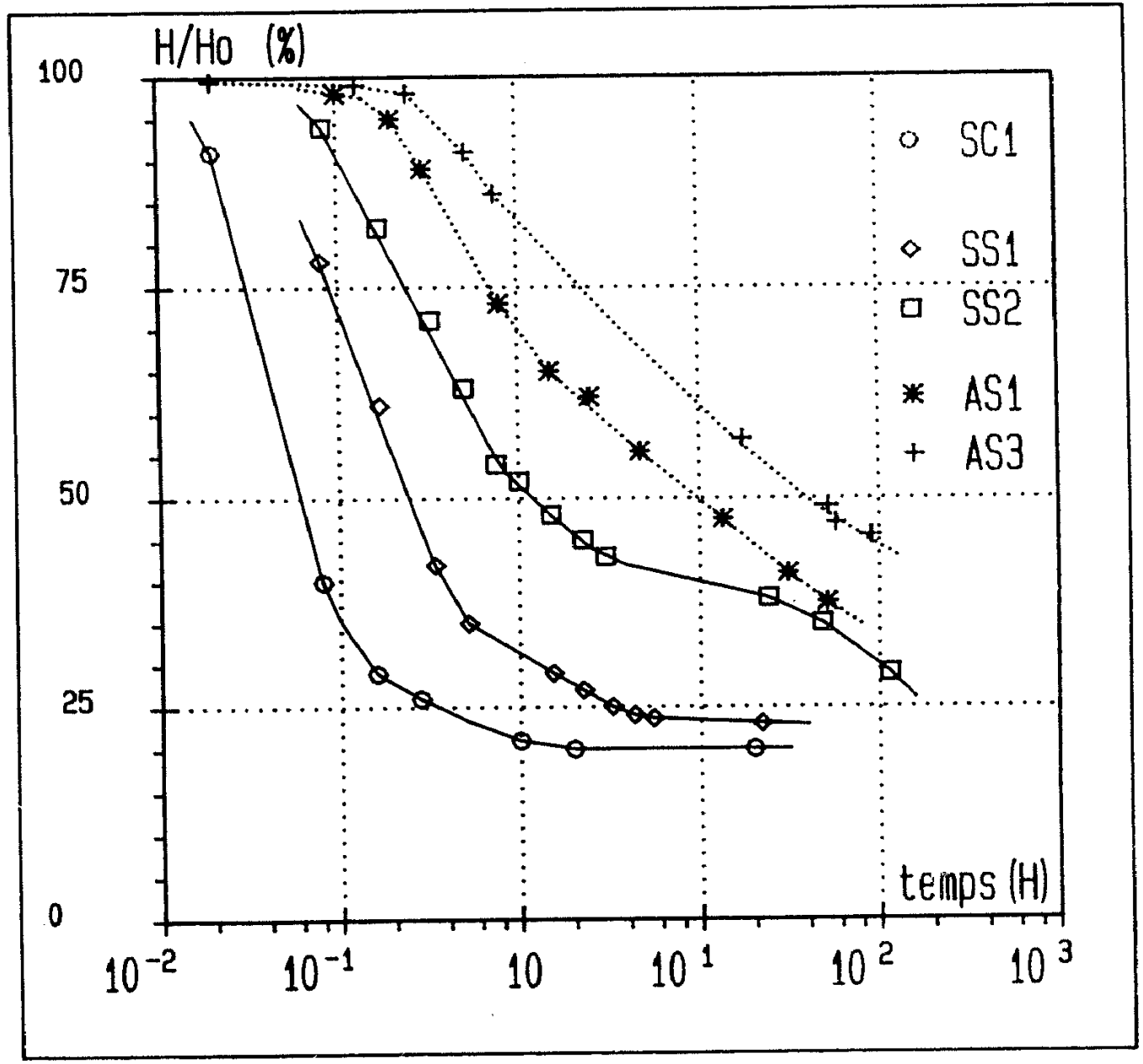

Fig. 3. - Courbes de tassement.

Fig. 3. - Settling curves. 


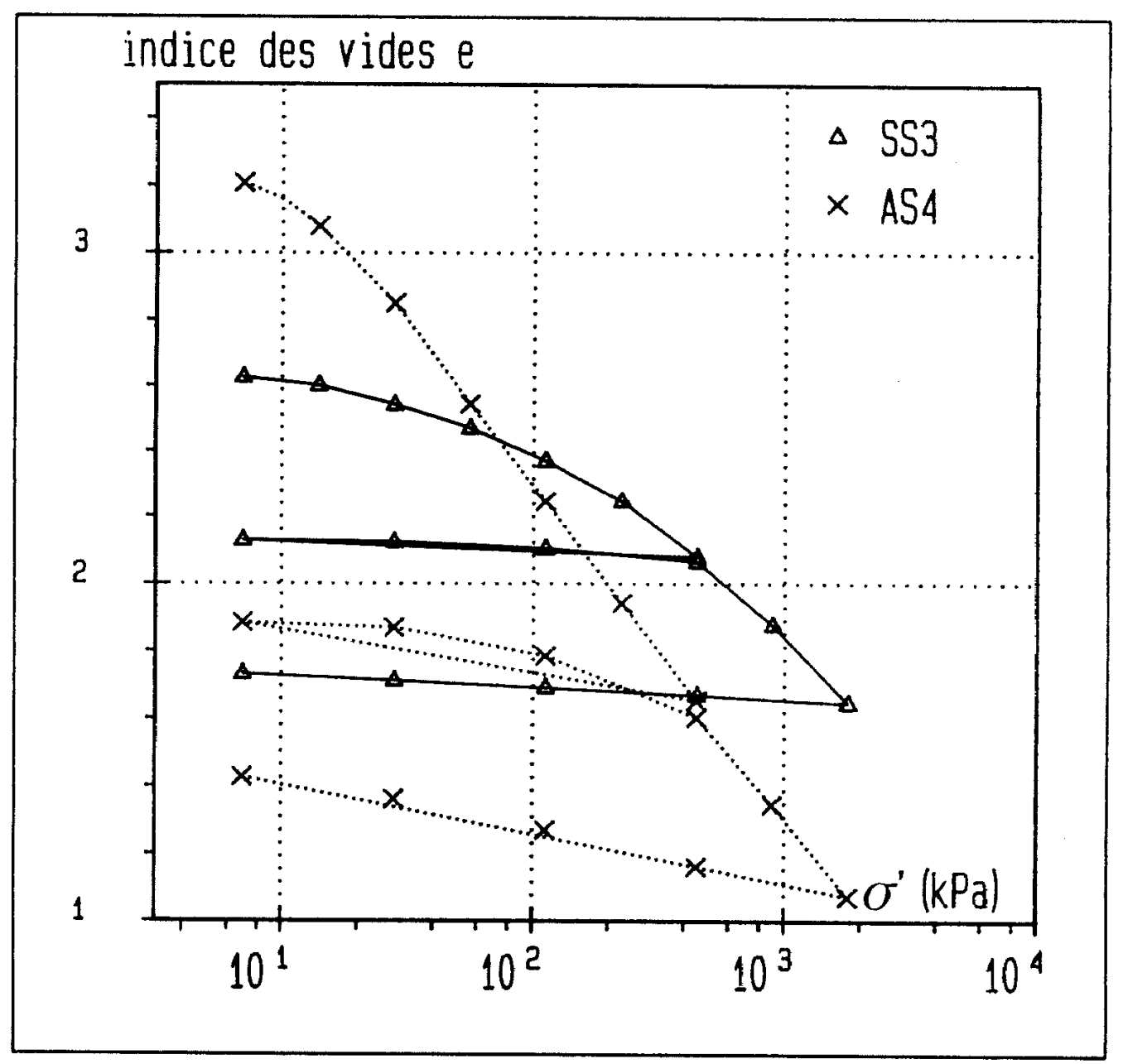

Fig. 4. - Courbes cedométriques.

Fig. 4. - œdometric curves.

\section{COMPORTEMENT ACOUSTIQUE DURANT LE TASSEMENT}

Dans les sédiments sableux, presque exclusivement formés de tests de foraminifères entiers à matrice quasi inexistante, la mesure de la vitesse du son n'a pu être mesurée qu'en fin de sédimentation. Elle est de l'ordre de $1525 \mathrm{~m} / \mathrm{s}$, légèrement supérieure à celle dans l'eau de mer. Les matériaux de type sableux acquièrent une structure rigide dès leur dépôt.

Pour les échantillons silteux ou argileux, une légère diminution de la vitesse du son apparaît lors de la sédimentation. Ce phénomène est particulièrement bien représenté pour l'échantillon SS2 et se développe en trois phases (fig. 5a)

- la vitesse du son est voisine de celle dans l'eau de mer $\left(V_{L}=1500 \mathrm{~m} / \mathrm{s}\right)$ : la solution est diluée et la propagation des ondes se fait dans l'eau. La présence de particules solides a peu d'influence;

- la vitesse du son diminue de façon plus marquée : la formation d'un complexe eau-particule entrave la propagation des ondes;

- dans le dépôt stabilisé, la vitesse du son devient constante. Inférieure à celle dans l'eau, elle varie de 1460 à $1480 \mathrm{~m} / \mathrm{s}$.

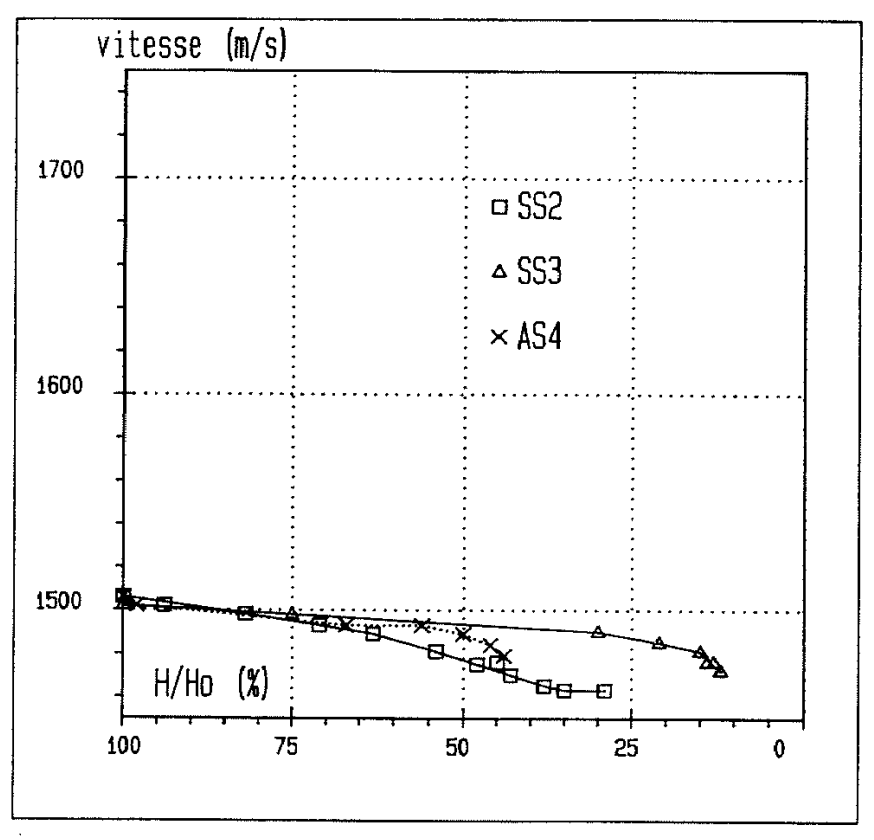

Fig. 5. - Vitesse du son en fonction du tassement. a. Phase de sédimentation.

Fig. 5. - Sound velocity versus settling. a. Decantation stage. 
Sous charge croissante, la célérité des ondes acoustiques s'accroît régulièrement de l'application de la contrainte à la stabilisation du tassement. La contrainte effective contrôle le rapprochement des particules et l'augmentation des contacts intergranulaires. L'allure des courbes vitesse-contrainte rappelle celle des courbes œedométriques (fig. $5 b$ et fig. 4); toutefois les courbes "vitesse " des silts carbonatés (SS3) et des argiles (AS4) sont très proches et moins typiques des sédiments que les courbes œdométriques. Elles se décomposent en trois parties ou paliers (fig. 5):

- un palier initial à faible variation de la vitesse du son $\left(\mathrm{V}_{\mathrm{L}} \approx 1500 \mathrm{~m} / \mathrm{s}\right)$;

- à partir d'une certaine valeur de la contrainte $\left(\sigma_{s}^{\prime}\right)$, la vitesse s'accroît nettement. $\sigma_{s}^{\prime}$ matérialise un changement d'état du sédiment;

- pour les charges plus élevées, la croissance des vitesses devient plus rapide et proportionnelle au logarithme de la contrainte.

Les deux phases successives de l'évolution de la vitesse des ondes dans l'échantillon argileux AS4 marquent deux phénomènes distincts qui se produisent simultanément mais avec prépondérance de l'un puis de l'autre : réarrangement des particules, ensuite leur rapprochement par expulsion de l'eau ce qui accroît les contacts entre les grains et par suite la vitesse du son. Pour les silts carbonatés SS3, le passage entre ces deux phases est progressif et traduit des processus de réorganisation des grains plus importants. Les valeurs de $\sigma_{s}^{\prime}$ correspondant au début du phénomène de réarrangement sont comprises entre 15 et $80 \mathrm{kPa}$, suivant la nature du matériau.

Ces résultats concordent avec les expériences de O'BRIEN (1963) et MARTIN (1965) (dans RIECKE et CHILINGARIAN, 1974) qui observent dès $100 \mathrm{kPa}$ une orientation préférentielle des particules.

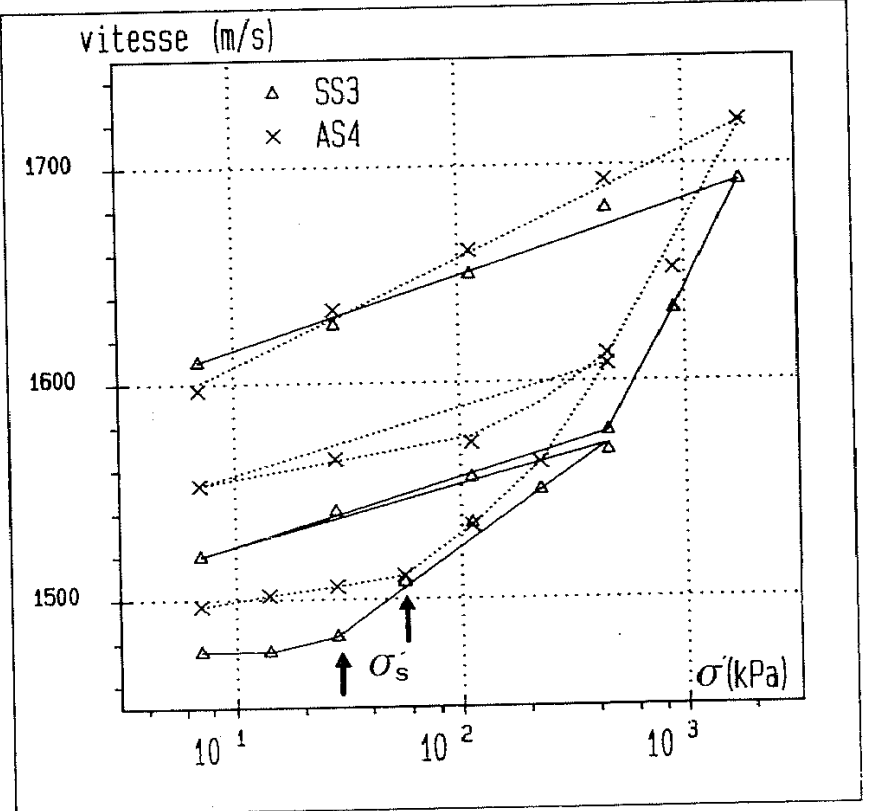

Fig. 5. - Vitesse du son en fonction du tassement. b. Phase de compaction.

Fig. 5. - Sound velocity versus settling. b. Compaction stage.
L'augmentation de la charge simulant l'enfouissement, les résultats précédents donnent aussi la valeur du gradient vitesse du son sur profondeur pour les sols étudiés (tab. 2). Alors que les gradients obtenus expérimentalement pour les matériaux argileux sont semblables à ceux mesurées in situ, pour les matériaux carbonatés ils sont plus faibles (MILHOLLAND et al., 1980 ; HAMILTON, 1985) ; précisons que dans notre étude nous ne tenons pas compte du facteur temps ni des phénomènes éventuels de diagenèse qui peuvent affecter ces formations (cimentation, recristallisation...).

Tableau 2. - Corrélations entre la vitesse du son et la profondeur d'enfouissement.

\begin{tabular}{|l|l|}
\hline $\begin{array}{l}\text { sédiment sableux } \\
\text { carbonaté }\end{array}$ & $V_{\mathrm{L}}(\mathrm{m} / \mathrm{s})=1532+0,70 \cdot \mathrm{Z}(\mathrm{m})$ \\
\hline $\begin{array}{l}\text { sédiments silteux } \\
\text { carbonatés }\end{array}$ & $\mathrm{V}_{\mathrm{L}}(\mathrm{m} / \mathrm{s})=1502+0,70 \cdot \mathrm{Z}(\mathrm{m})$ \\
\hline sédiments argileux & $\mathrm{V}_{\mathrm{L}}(\mathrm{m} / \mathrm{s})=1510+1,04 \cdot \mathrm{Z}(\mathrm{m})$ \\
\hline
\end{tabular}

\section{VITESSE DU SON ET POROSITÉ}

Lors de l'accroissement de la contrainte, nous suivons la diminution des vides par la variation de la porosité (fig. 6). La courbe de chargement de l'échantillon argileux (AS4) fait apparaitre deux segments linéaires : pour les fortes porosités la vitesse augmente très légèrement quand la porosité diminue, pour des plus faibles porosités la vitesse s'accroît fortement. A chaque partie de la courbe correspond une relation linéaire de la forme : $\mathrm{V}_{\mathrm{L}}=\mathrm{a} \cdot \mathrm{n}+\mathrm{b}$ ( $\mathrm{a}$ et $\mathrm{b}$ étant des paramètres dépendant de la composition du sédiment). Le passage entre les deux tronçons détermine une valeur de porosité seuil $\left(n_{\mathrm{s}}\right)$ caractéristique du

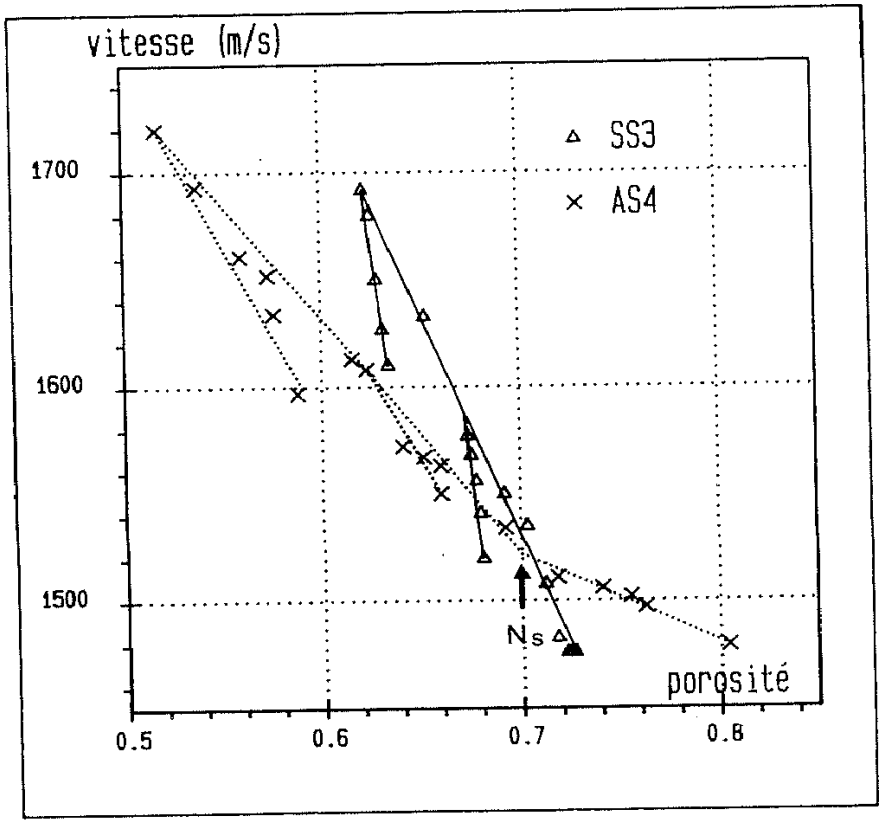

Fig. 6. - Vitesse du son en fonction de la porosité. Fig. 6. - Sound velocity versus porosity. 
matériau. Elle correspond à la contrainte seuil déterminée précédemment. Dans l'échantillon silteux carbonaté (SS3) seule la partie à moindre porosité est présente et la porosité seuil est assimilable à la porosité initiale.

Cette valeur est à comparer aux valeurs classiques de changement d'état:

- la limite de liquidité d'Atterberg;

- la transition vase-floculat habituellement prise pour le minimum de la courbe de WOOD (MIZIKOS, 1972).

Pour les échantillons argileux les valeurs de porosité seuil $\left(\mathrm{n}_{\mathrm{s}}\right.$ de 0,66 à 0,72$)$ sont légèrement inférieures à celles correspondant aux limites de liquidité $\left(\mathrm{n}_{\mathrm{L}}\right.$ de $0,72$ à 0,76$)$. Pour les échantillons silteux carbonatés $\left(\mathrm{n}_{\mathrm{s}} \approx 0,72\right)$, la valeur de limite de liquidité est plus faible $\left(\mathrm{n}_{\mathrm{L}} \approx 0,64\right)$; mais les limites d'Atterberg sont peu adaptées à ce type de sédiment grossier. Dans tous les cas les valeurs de $n_{5}$ se situent en dessous du minimum de la courbe de WOOD ( $\mathrm{n}$ de 0,75 à 0,80 ). Ceci nous permet d'affiner la détermination du passage des comportements liquide à plastique et de préciser l'acquisition d'une structure rigide.

L'augmentation de la contrainte implique bien sûr une diminution de la porosité, mais surtout une réorganisation du réseau des particules. Pendant la compaction, les grains s'orientent perpendiculairement à la pression, une structure de type désordonnée devient une structure de type orientée (MEADE, 1966 ; BENNETT et al., 1981). Il en résulte un changement dans la géométrie des pores (distribution et orientation spatiale) qui se répercute sur la propagation des ondes acoustiques (KIM et al., 1985)

Lors de la phase de déchargement la diminution de vitesse est plus importante que la diminution de porosité pour les deux matériaux. La relation vitesseporosité n'est donc pas réversible, en raison de l'augmentation de contrainte. Pour chaque déchargement, la relation est de type linéaire, la pente de la courbe est identique, mais l'origine est augmentée d'une constante $c$ due à l'accroissement de la contrainte: $V_{L}$ $=a^{\prime} \cdot n+b^{\prime}+c$ (a' et b' étant deux paramètres dépendant de la composition du sédiment et $\mathrm{c}$ un paramètre lié à la contrainte appliquée).

Les sédiments silteux, composés d'éléments hétérogènes aux formes variées (tests et débris carbonatés), présentent lors de la compaction un réarrangement irréversible des particules, lié à leur orientation, leur déplacement et parfois même leur rupture. Ces processus augmentent le nombre des contacts entre les grains donc la vitesse du son. La présence de minéraux argileux, formant un film autour des particules, accroît la surface de réaction. Lors de la décharge, les contacts entre les grains se relâchent provoquant ainsi une diminution de la vitesse du son mais sans modifier notablement la porosité.

Les matériaux argileux sont composés essentiellement de plaquettes et de feuillets à grande surface spécifique. La destruction des structures de dépôt et le début d'orientation des particules intervient dans les premiers temps de la compaction. A partir de la contrainte seuil $\left(\sigma_{s}^{\prime} \approx 100 \mathrm{kPa}\right)$, le tassement se fait par rapprochement des feuillets. Le gonflement de ces échantillons se traduit par un relâchement du réseau et un écartement des feuillets donc une augmentation de la porosité. Dans ce cas la relation vitesseporosité devient presque réversible.

L'évolution de la vitesse du son en fonction de la porosité pour la totalité des échantillons est comparée aux courbes théoriques de WOOD, de WYLLIE et de NOBES (fig. 7). Les valeurs des paramètres utilisés pour le calcul de la courbe de WOOD sont:

$$
\begin{aligned}
& \rho_{\mathrm{s}}=2,56 \mathrm{t} / \mathrm{m}^{3} ; \rho_{\mathrm{w}}=1,03 \mathrm{t} / \mathrm{m}^{3} ; \mathrm{k}_{\mathrm{s}}= \\
& 1,22.10^{-11} \mathrm{~m}^{2} / \mathrm{N} ; \mathrm{k}_{\mathrm{w}}=4,3 \cdot 10^{-10} \mathrm{~m}^{2} / \mathrm{N}\left(\mathrm{V}_{\text {eau }}\right. \\
& =1500 \mathrm{~m} / \mathrm{s}) .
\end{aligned}
$$

Les points des échantillons argileux se localisent entre les courbes des modèles de WOOD et de NOBES, ces sédiments présentent un comportement typique de suspensions possédant déjà une certaine rigidité (fig. $7 a)$. Leurs valeurs de porosité seuil sont comprises entre 0,66 et 0,72 . L'équation de lissage est de la forme: $V_{L}(m / s)=1600 n^{2}-2870 n+2750$ proche de celle des sédiments du bassin brésilien (ORSI et DUNN, 1989): $V_{1}(\mathrm{~m} / \mathrm{s})=1782 \mathrm{n}^{2}-$ $2713 \mathrm{n}+2527$.

Par contre l'évolution des silts carbonatés est différente et leur comportement se rapproche de celui des milieux poreux du modèle de WYLLIE (fig. 7b). D'une manière générale, la vitesse du son est plus élevée dans les matériaux grossiers et carbonatés. La valeur de porosité seuil est inférieure ou égale à 0,72 . Dans notre domaine d'étude, nous obtenons la relation linéaire moyenne

$$
\mathrm{V}_{\mathrm{L}}(\mathrm{m} / \mathrm{s})=2975-2090 \mathrm{n} \text {. }
$$

Bien que la vitesse du son dans les carbonates et les silicates soit plus élevée que dans les argiles $\left(\mathrm{V}_{\mathrm{L}}\right.$ calcite $\approx 6600 \mathrm{~m} / \mathrm{s}, V_{\mathrm{L}}$ quartz $\approx 6000 \mathrm{~m} / \mathrm{s}, \mathrm{V}_{\mathrm{L}}$ argiles $\approx 3400 \mathrm{~m} / \mathrm{s}$ ), la composition chimique seule ne permet pas d'expliquer la différence de comportement acoustique entre les deux types de matériaux. Il s'agit essentiellement de l'influence de la granulométrie qui transparaît. Les études réalisées sur divers sédiments naturels ont donné de bonnes corrélations entre les paramètres grain moyen et pourcentage de grossiers sur la vitesse du son (GREFFARD, 1969 ; HAMILTON et al., 1982 ; BACHMAN, 1985). La relation vitesse-teneur en carbonate parfois observée est en grande partie due à la nature en majorité calcaire de la fraction grossière.

Par ailleurs selon HAMILTON et al. (1982), la présence de tests creux de foraminifères influe sur la relation célérité du son-porosité. En effet, l'eau intraparticulaire ne joue aucun rôle dans la propagation des ondes, par contre elle augmente artificiellement la porosité du sédiment. Ce phénomène est particulièrement visible pour l'échantillon SC1 dont le point à plus faible porosité semble se rapprocher de la tendance générale. Il s'agit, en fait, de la diminution de la porosité intraparticulaire due à la rupture de particules carbonatées observée durant l'essai œedométrique. 


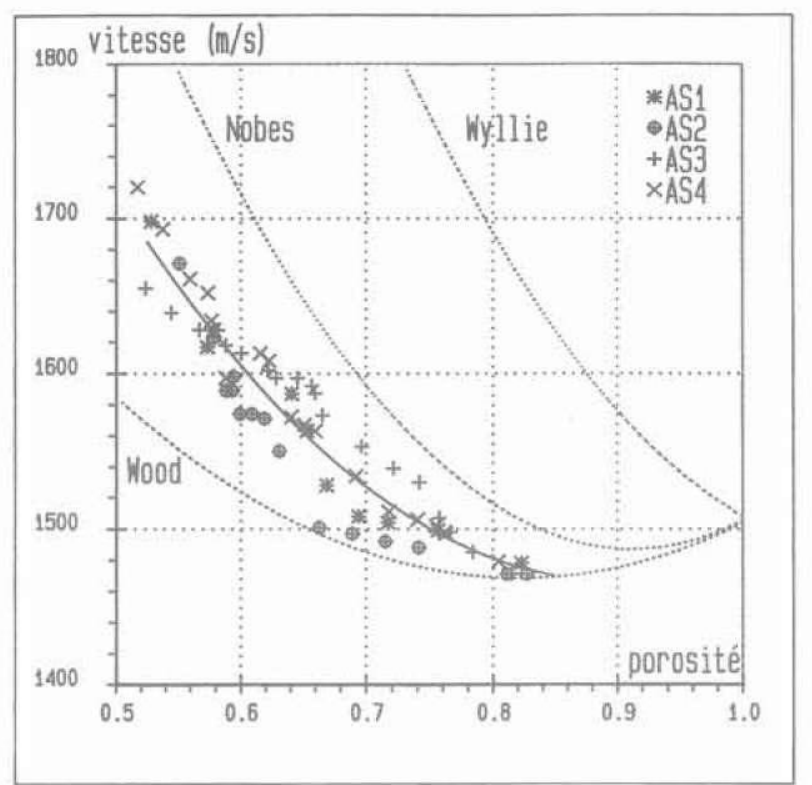

Fig. 7. - Diagramme vitesse du son-porosité : a. Sédiments argileux. b. Sédiments carbonatés. Fig. 7. - Graph sound velocity versus porosity: a. Argileous sediments. b. Calcareous sediments.

\section{CONCLUSION}

La mesure de la vitesse du son durant les premiers stades du tassement expérimental de sédiments marins permet de suivre sous charge les relations entre la phase solide et le liquide. Le changement d'état des matériaux apparaît dès les faibles contraintes (inférieures à $100 \mathrm{kPa}$ ) et traduit l'acquisition de la cohésion et de la rigidité résultant du réarrangement des particules.

Cette étude confirme pour les sédiments argileux la relation vitesse du son-porosité : diminution de la vitesse du son lors de la sédimentation puis augmentation lors de la compaction (modèles de WOOD et de NOBES). Par contre, pour les silts sableux carbonatés l'accroissement de vitesse est plus rapide en raison d'une granulométrie plus grossière.

\section{BIBLIOGRAPHIE}

BACHMAN R.T. (1985), Acoustic and physical property relationship in marine sediment. J. Acoust. Soc. Am., vol. 78, $n^{\circ} 22,1985$, p. 616-621.

BENNET R.H., BRYANT W.R., KELLER G.H. (1981), Clay fabric of selected submarine sediments : fundamental properties and models. J. Sed. Pet., vol. 51, n० 1, p. 217-232.

GREFFARD J. (1969), Contribution à l'étude des relations entre la propagation du son et la nature physico-chimique des sédiments marins. Cahier Océan., Paris XXle année, n² 2, p. 145-171.

GUILLAUME P. (1990), Etude des caractéristiques géotechniques de sols sous-marins du Domaine Caraỉbe : rapports à la sédimentogenèse et aux facteurs géochimiques. Thèse de l'Université de Bordeaux 1, 1990, 258 p.

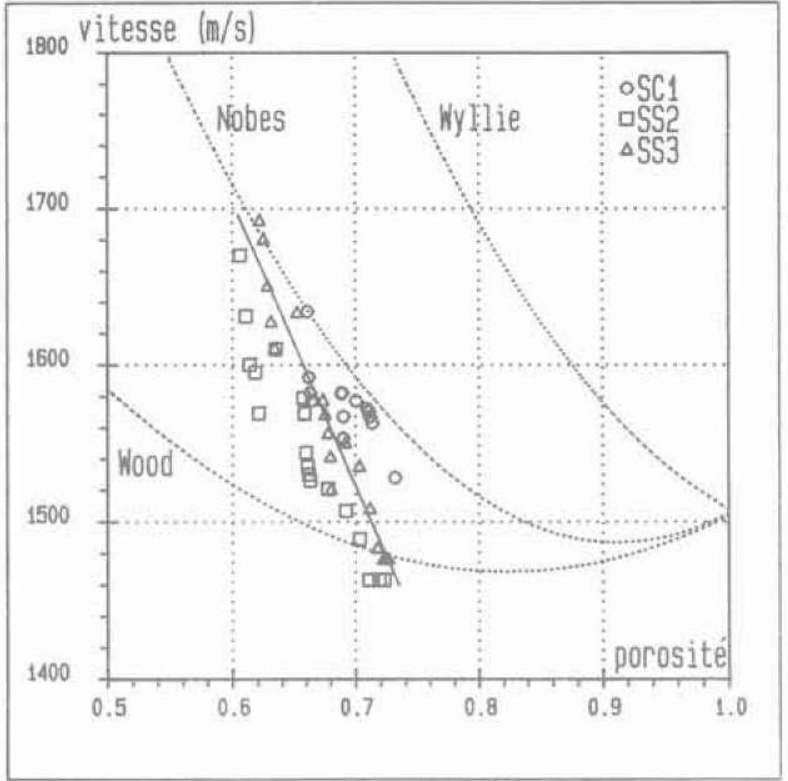

HAMILTON E.L. (1985), Sound velocity as a fonction of depth in marine sediments. J. Acoust. Soc. Am., vol. 78, n 4, 1985, p. 1348-1335.

HAMILTON E.L. et al. (1982), Acoustic and related properties of calcareous deep-sea sediments. J. Sed. Pet., vol. 52, n³, 1982, p.. 733-753.

KIM D.C., MANGHNANI M.H., SCHLANGER S. (1985), The role of diagenesis in the development of phusical properties of deep-sea carbonate sediments. Mar. Geol., vol. 69, 1985, p. 69-91.

LAUGHTON A.S. (1957), Soun propagation in compacted ocean sediments. Geophysics, vol. 22 , $\mathrm{n}^{\circ} 2,1957$, p. 233-260.

MARTIN R.T. (1965), Quantitative measurements of wet clay fabric. North Am. Clay Miner. Conf., 14th, Berkeley, 1965, 31 p.

MEADE R.H. (1986), Factors influencing the early stages of the compaction of clays and sandsreview. J. Sed. Pet., vol. 36, $\mathrm{n}^{\circ} 4,1966, \mathrm{p}$. 1085-1101.

MIGNIOT C. (1968), Etude des propriétés physiques de différents sédiments très fins et leur comportement sous des actions hydrodynamiques. La Houille Blanche, $\mathrm{n}^{\circ}$ spécial 7, 1968, p. 591-620.

MILHOLLAND P.D. et al. (1980), Geoacoustic modeling of deep-sea carbonate sediments. J. Acoust. Soc. Am., vol. 68, 1980, p. 1351-1360.

MIZIKOS J.P. (1972), Propriétés mécaniques et physiques des sédiments marins meubles. Thèse doct. ès Sciences, Nice, 1972, 203 p.

NOBES D.C. (1989), A test of a simple model of the acoustic velocity in marine sediment. J. Acoust. Soc. Am., vol. 86, $\mathrm{n}^{\circ} 1,1989$, p. 291-294.

O'BRIEN N.R. (1963), A study of fissility in argillaceous rocks. $\mathrm{Ph}$. D. Thesis, Univ. Illinois, Urbana, 1963, $80 \mathrm{p}$. 
ORSI T.H., DUNN D.A. (1990), Sound velocity et related properties of fine-grained abyssal sediments from the Brazil Basin (South Atlantic Ocean). J. Acoust. Soc. Am., vol. 88, n 3, 1990, p. 1536-1542.

RIEKE H.H., CHILINGARIAN G.V. (1974), Compaction of argilous sediments. Developments in Sedimentology $\mathrm{n}^{\circ} 16$. Elsevier, 1974, $424 \mathrm{p}$.

SHUMWAY G. (1960), Sound speed and absorption studies of marine sediment by resonance method.
Part. I : Geophysics, vol. 25, n², 1960, p. 451-467. Part II : Geophysics, vol. 25, n³, 1960 , p. 659-692.

WOOD A.B. (1941), A text book of sound. G. Belle and sons, London, 1941.

WYLLIE M.R., GARDNER G.H., GREGORY A.R. (1962), Studies of elastic wave attenuation in porous media. Geophysics, vol. 27, 1962, p. 569-589. 